\title{
Umsetzungen von Cyclopropyllithium mit Halogenbenzolen
}

\author{
Walter Kurtz ${ }^{1)}$ und Franz Effenberger *
}

Institut für Organische Chemie der Universität Stuttgart, D-7000 Stuttgart 1, Azenbergstr. 14

Eingegangen am 28. August 1972

Cyclopropyllithium und Halogenbenzole $(\mathbf{1}, \mathbf{5}, \mathbf{1 1})$ ergeben beim Erhitzen in siedendem $\ddot{A}$ ther Cyclopropylbenzole und Biphenyle; daneben findet Enthalogenierung statt. Die Produktverteilung erlaubt Schlüssc auf den Mechanismus dieser Reaktionen: Für die Enthalogenierung der Bromaromaten wird ein Metall-Halogen-Austausch im üblichen Sinne, bei den Fluor- und Chlorverbindungen Arinbildung und anschließende Hydridübertragung angenommen. Die Einführung von Cyclopropylresten in die Aromaten und dic Biarylbildung können sowohl durch direkte nucleophile Substitution wie über Arin-Zwischenstufen erfolgen. Zunehmende Cyclopropylsubstitution im $\Lambda$ romaten erschwert die Metallierung und damit dic Arinbildung; dagegen wird die Direktsubstitution aufgrund der besseren Stabilisierung der anionischen $\sigma$-Komplex-Zwischenstufe begünstigt.

\section{Reaction of Cyclopropyllithium with Halobenzenes}

Cyclopropyllithium and halobenzenes $(\mathbf{1}, \mathbf{5}, \mathbf{1 1})$ afford cyclopropylbenzenes and biphenyls upon refluxing in ether; in addition considerable dehalogenation takes place. From the composition of the product mixture, conclusions can be drawn as to the mechanism of these three types of reaction. Bromobenzenes are supposed to be dehalogenated by metal-halogen interconversion in the usual way, while for fuoro and chloro compounds benzyne formation followed by hydride transfer is held more likely. Both introduction of cyclopropyl groups into the arene nucleus and biaryl formation may proceed by direct nucleophilic substitution as well as via benzyne intermediates. Introduction of more cyclopropyl substituents into the arene nucleus decreases the rate of metalation and thus aryne formation; direct nucleophilic substitution, on the other hand, hecomes more favoured due to increasing stabilization of the anionic $\sigma$-complex intermediate.

Wie wir vorstehend berichtet haben 2), lassen sich Polycyclopropylbenzole mit zueinander $m$-ständigen Substituenten in brauchbaren Ausbeuten nur durch Cyclopropanierung der entsprechenden Polyolefine nach dem Makosza-WawrzyniewiczVerfahren ${ }^{3)}$ darstellen. Wegen der ausgeprägt o/p-dirigierenden Wirkung des Dreirings ist die Synthese von 1,3-Di- und 1,3,5-Tricyclopropylbenzol aus Cyclopropylbenzol über eine elektrophile Einführung geeigneter Substituenten, die sich in Cyclopropyl überführen lassen, direkt nicht möglich. 1-Halogen-4-cyclopropyl- und 1-Halogen-2,4-dicyclopropylbenzole dagegen sind auf diese Weise leicht zugänglich4), so daß es nahe lag zu versuchen, hieraus über Arinreaktionen mit Cyclopropyllithium zu den gewünschten Verbindungen zu gelangen. Die direkte nucleophile Einführung von

1) Teil der Dissertation W. Kurtz, Univ. Stuttgart 1971.

2) F. Effenberger und $W$. Kurtz, Chem. Ber. 106, 511 (1973).

3) M. Makosza und W. Wawrzyniewicz, Tetrahedron Letters [London] 1969, 4659.

4) W. Kurtz, P. Fischer und F. Effenberger, Chem. Ber. 106, 525 (1973). 
Cyclopropylgruppen in aromatische Systeme würde einen weiteren, präparativ allgemein anwendbaren Zugang zu Cyclopropylaromaten darstellen. Da diese Synthesemöglichkeit unseres Wissens bislang in der Literatur nicht beschrieben ist, haben wir die Reaktion von Cyclopropyllithium mit Halogenaromaten eingehender untersucht. Aufgrund der sp2-Hybridisierung der Ring-C-Atome ist das anionische Zentrum im Cyclopropyllithium vergleichsweise weniger nucleophil als bei normalen sekundären Alkyllithiumverbindungen ${ }^{5)}$. Die Reaktivität von Cyclopropyllithium gegenüber Diäthyläther ist daher nur gering $\left(t_{1 / 2} \quad 140 \mathrm{~h}^{6}\right)$ ), etwa in der Größenordnung von n-Butyllithium, so daß die Umsetzungen mit Halogenbenzolen nicht bei tiefer Temperatur durchgeführt werden müssen.

\section{Umsetzungen von Cyclopropyllithium mit Halogenbenzolen}

Eine aus Cyclopropylbromid und Lithium in absol. Äther bei $0^{\circ}$ jeweils frisch bereitete Cyclopropyllithium-Lösung wird in etwa vierfachem Überschuß vorgelegt, das Halogenbenzol zugegeben und anschließend unter Rückfluß erhitzt. Die Produkte werden durch Vergleich der Retentionszeiten mit den Werten für unabhängig synthetisierte Vergleichssubstanzen und durch kombinierte Gaschromatographie-Massenspektroskopie 7) identifiziert.

Die Umsetzung der Halogenbenzole la, b mit Cyclopropyllithium liefert Benzol, Cyclopropylbenzol (3) und 2-Cyclopropylbiphenyl (4); bei 1c entsteht außerdem noch Biphenyl (Tab, 1).

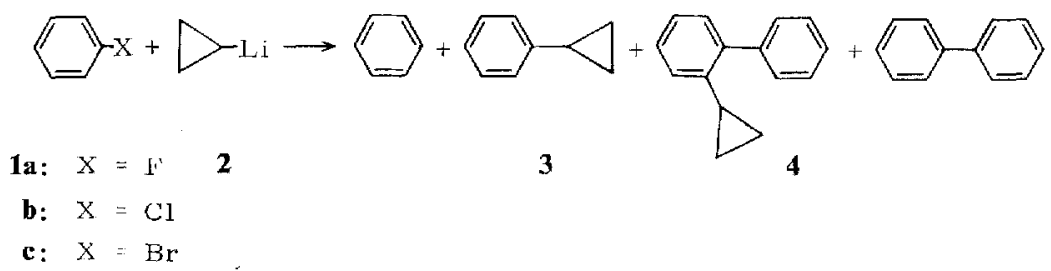

Bei der Reaktion der $p$-Halogen-cyclopropylbenzole 5 ist das Produktspektrum erwartungsgemäß breiter, wobei die Cyclopropylbenzole 3, 6, 7 und 4,4'-Dicyclopropylbiphenyl (8) eindeutig charakterisiert werden konnten. Daneben ließen sich noch zwei isomere Tricyclopropylbiphenyle nachweisen, denen aufgrund mechanistischer Überlegungen (s. u.) die Strukturen 9 und 10 zukommen.

Aus 1-Chlor- und 1-Brom-2,4-dicyclopropylbenzol (11 b, c) entstehen neben dem Enthalogenierungsprodukt 6 die Tricyclopropylbenzole 12 und 13 sowie zu etwa $4 \%$ Polycyclopropylbiphenyle, deren Struktur nicht genau ermittelt wurde (Tab. 1 ).

Wie die Umsetzungen von 1, 5 und 11 mit 2 zeigen, ist es prinzipiell möglich, Cyclopropylgruppen über eine nucleophile Substitution in Aromaten einzuführen; die Produktverteilung bei den einzelnen Reaktionen ist in Tab. 1 nach Reaktionstypen

5) D.J. Cram, Fundamentals of Carbanion Chemistry, S. 49, Academic Press, New York und London 1965.

6) D. Seyferth und H. M. Cohen, J. organomet. Chem. 1, 15 (1963).

7) Für die GC-MS-Aufnahmen sind wir Herrn $W$. Blum, Finnigan GmbH, München, zu Dank verpflichtet. 

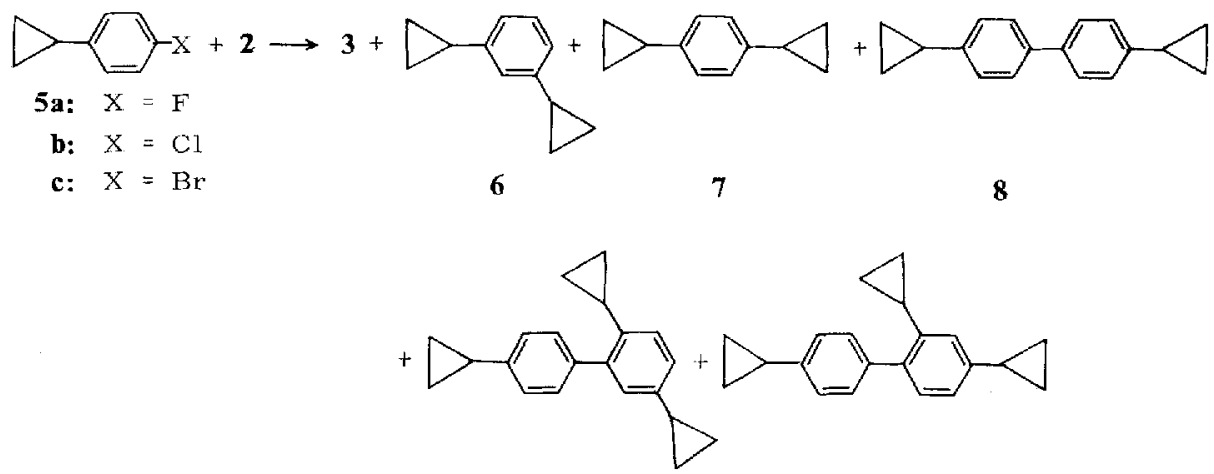

9

10

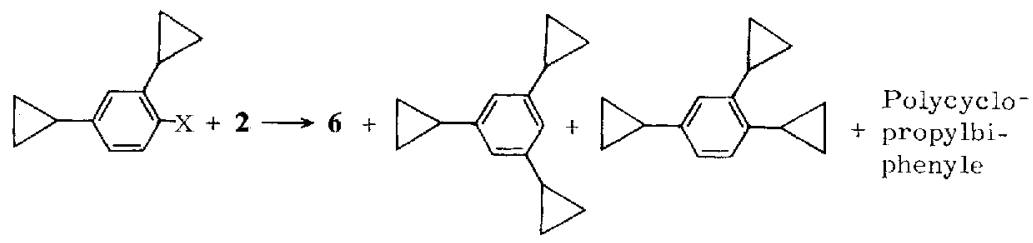

$\begin{aligned} \text { 11b: } & X=C 1 \\ \text { c: } & X=B r\end{aligned}$

12

13

zusammengefaßt. Der alternative Syntheseweg ist ebenso gangbar: aus Phenyllithium und Cyclopropylbromid erhält man 3 mit $45 \%$ Ausbeute, bezogen auf eingesetztes $\mathrm{C}_{3} \mathrm{H}_{5} \mathrm{Br}$.

Tab. 1. Produktspektrum bei der Umsetzung der Halogenbenzole 1, 5 und 11 mit Cyclopropyllithium (gaschromatographisch bestimmter Anteil am isolierten Produktgemisch)

\begin{tabular}{|c|c|c|c|c|c|}
\hline \multirow{2}{*}{$\begin{array}{l}\text { Einge- } \\
\text { setztes } \\
\text { Halogen- } \\
\text { benzol }\end{array}$} & \multirow{2}{*}{$\begin{array}{c}\text { Reakt.- } \\
\text { Zeit } \\
\text { (h) }\end{array}$} & \multicolumn{4}{|c|}{ Erhaltene Produkte $(\%)$} \\
\hline & & $\begin{array}{c}\text { Aus- } \\
\text { gangs- } \\
\text { produkt }\end{array}$ & $\begin{array}{l}\text { Enthalo- } \\
\text { genierung }\end{array}$ & $\begin{array}{c}\text { Cyclo- } \\
\text { propanierung }\end{array}$ & Biphenyle \\
\hline $\mathbf{1 a}$ & 12 & - & Benzola) & $3(58)$ & $4(34)$ \\
\hline $\mathbf{1 b}$ & 80 & - & Benzol a) & $3(82)$ & $4(11)$ \\
\hline $1 \mathrm{c}$ & 58 & - & Benzol a) & $3(58)$ & 4 (12), Biphenyl (11) \\
\hline $\mathbf{5 a}$ & 1.2 & 43 & $3(16)$ & $6(16), 7(14)$ & $9 \div 10 \quad(9)$ \\
\hline $5 \mathbf{b}$ & 1.2 & 88 & $3(3)$ & $6 \quad(3), 7 \quad(3)$ & - \\
\hline $5 \mathbf{c}$ & 1.2 & 55 & $3(40)$ & $6 \quad(2), 7 \quad(3)$ & $\ldots$ \\
\hline $5 \mathbf{a}$ & 12 & - & $3(34)$ & $6(28), 7(25)$ & $9+10(13)$ \\
\hline $5 \mathrm{~b}$ & 85 & 8 & $3(35)$ & $6(22), 7(21)$ & $9+10(9)$ \\
\hline $5 c$ & 12 & $\cdot-$ & $3(66)$ & $6(4), 7(19)$ & $9+10(2), 8(2)$ \\
\hline $11 \mathrm{~b}$ & 90 & 26 & $6(30)$ & $12(27)$ & \\
\hline $11 \mathrm{c}$ & 12 & 4 & $6(89)$ & $12(3), 13(4)$ & -- \\
\hline
\end{tabular}

a) Bei der gewählten Aufarbeitungsmethode läßt sich der Anteil des gebildeten Benzols nicht oxakt bestimmen; bei Rückrechnung auf eingesetztes Halogenbenzol ergeben sich für 3 folgende Prozentwerte: 1 a $42 \%$, 1b $58 \%, 1$ c $29 \%$. 


\section{Diskussion der Produktbildung}

Als starke Base und starkes Nucleophil kann Cyclopropyllithium (2) mit Halogenbenzolen über Dehydrobenzol-Zwischenstufen (Weg A) oder in einer direkten nucleophilen Substitution (Weg B) zu Cyclopropylbenzolen reagieren.

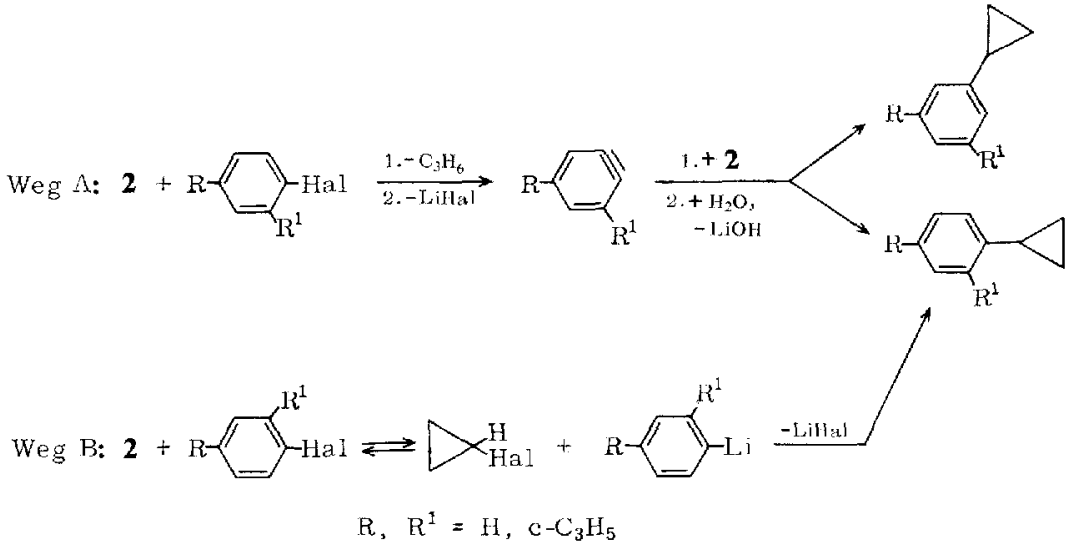

Wie in vergleichbaren Systemen ${ }^{8}$ ist für 2 und Halogenbenzole Metall-HalogenAustausch im Sinne des formulierten Gleichgewichts anzunehmen; daher kann die Direktsubstitution einerseits als nucleophiler Angriff von 2 am Halogenbenzol, zum andern durch Reaktion des Aryl-Anions mit dem Cyclopropylhalogenid erfolgen. Angriff des Aryl-Anions erscheint gesichert bei Alkylhalogeniden, die auch sonst normale nucleophile Substitution eingehen ${ }^{91}$; dagegen müssen bei den zumeist unter Umlagerung reagierenden Halogencyclopropanen beide Möglichkeiten in Betracht gezogen werden.

Ebenso läßt sich die Biphenylbildung über Arin-Zwischenstufen wie über Direktsubstitution deuten. Friedman und Chlebowski ${ }^{10}$ ) formulieren die Biarylverknüpfung bei Fluor-, Chlor- und Brombenzolen ausschließlich über primäre Arinbildung, während nach Winkler und Winkler ${ }^{8)}$ bei langen Reaktionszeiten 5-10\% Biaryl durch Direktsubstitution entstehen. Bei unseren Umsetzungen müssen Arinreaktion und Direktsubstitution nebeneinander ablaufen; zu diesem Schluß zwingen Substitutionstyp und relative Ausbeute der erhaltenen Cyclopropylbiphenyle. Im folgenden Schema sind alle Reaktionswege aufgezeigt, über die eine Aryl-Aryl-Verknüpfung erfolgen kann.

Metall-Halogen-Austausch ohne anschließende Folgereaktionen führt bei der hydrolytischen Aufarbeitung zu enthalogenierten Aromaten, wobei der hohe Anteil an Enthalogenierung bei den Fluor-Verbindungen durch den normalen Austauschmechanismus nicht zu erklären ist (s. u.).

8) H. J.S. Winkler und H. Winkler, J. Amer, chem. Soc. 88, 964, 969 (1966).

9) J. March, Advanced Organic Chemistry: Reactions, Mechanisms, and Structure, S. 354, McGraw-Hill Inc., New York 1968.

10) L. Friedman und J. F. Chlebowski, J. Amcr. chem. Soc. 91,4864 (1969). 
<smiles>[R]c1ccc(-c2ccccc2)cc1</smiles>

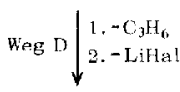

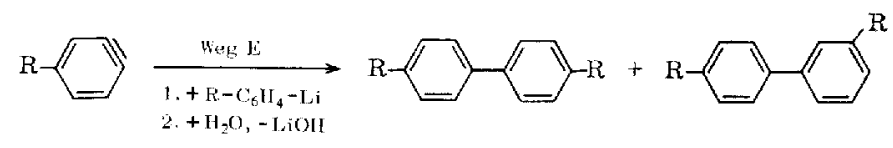
weg $F \downarrow+2$

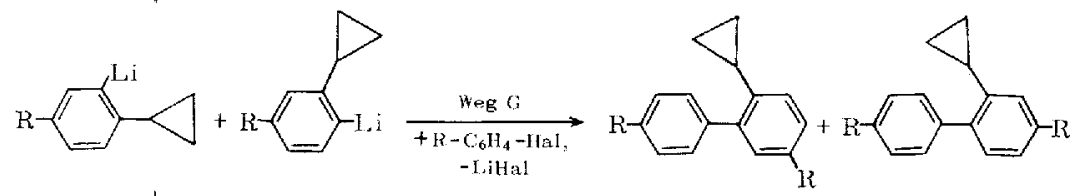

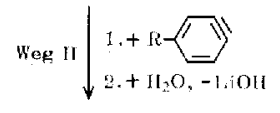

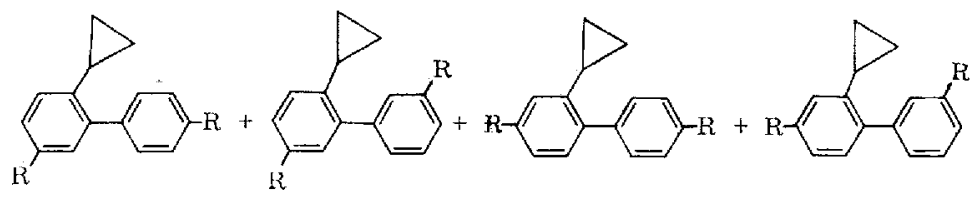

$$
\mathrm{R}=\mathrm{H}, \mathrm{c}-\mathrm{C}_{3} \mathrm{H}_{5}
$$

Anhand der jeweiligen Produktverteilung bei den Umsetzungen von 1, 5 bzw. 11 mit 2 (Tab. 1) sollen im folgenden die drei Grundreaktionen - Arinbildung, Direktsubstitution und Enthalogenierung - und ihre Beeinflussung durch Cyclopropylsubstituenten im einzelnen erörtert werden.

Enthalogenierung: Metall-Halogen-Austausch wird bei der Einwirkung von Organolithium-Verbindungen auf organische Halogenverbindungen vor allem dann in Konkurrenz zur Arinreaktion treten, wenn die Metallierung als deren geschwindigkeitsbestimmender Schritt erschwert ist. Die Acidität des zum Halogen o-ständigen Protons wird vermindert, wenn Substituenten mit + I-Effekt wie etwa Alkylgruppen die Elektronendichte auch im $\sigma$-Bindungsgerüst des Aromaten erhöhen. Damit wird die von 1c über 5c $(66 \%)$ zu 11 c $(89 \%)$ steigende Enthalogenierung leicht verständlich: mit zunehmender Substitution erhöht sich die Aktivierungsschwelle für die o-Metallierung und damit die Arinbildung, so daß die Austauschreaktion mehr und mehr zum Zuge kommen kann ${ }^{11)}$.

Bei den weniger elektronegativen Halogenen Brom und Jod ist die Acidität der $o$-Arylprotonen im Vergleich zu Fluor und Chlor ebenfalls stark verringert ${ }^{12)}$ und

11) I. Sauer, R. Huisgen und A. Hauser, Chem. Ber. 91, 1461 (1958).

12) R. G. Jones und H. Gilman, Org. Reactions 6, 339 (1951). 
damit die Austauschreaktion gegenüber der Metallierung relativ begünstigt. Für das System Jodbenzol/Cyclopropyllithium, bei dem praktisch keine irreversiblen Folgereaktionen beobachtet werden, können Applequist und $O^{\prime}$ Brien ${ }^{13)}$ daher eine Gleichgewichtskonstante $(K=: 9.55)$ angeben, wobei die Equilibrierung in $\ddot{A}$ ther selbst bei $-70^{\circ}$ noch rasch verläuft.

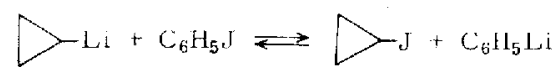

Bei unseren Verbindungen erfordert die Einstellung des Gleichgewichts offensichtlich mehrere Stunden, denn bei der Umsetzung von 5c mit 2 liegen nach $1.2 \mathrm{~h}$ erst $40 \%$ p-Cyclopropyl-phenyllithium neben $55 \%$ Ausgangsverbindung vor, während gegen Ende der Reaktion, entsprechend den Befunden bei Jodbenzol, kein 5c mehr nachzuweisen ist.

Der hohe Anteil an Enthalogenierung bei der Umsetzung von 1a und 5a fügt sich nicht in dieses Bild: unter gleichen Bedingungen (Reaktionszeit $1.2 \mathrm{~h}$ ) wird $5 \mathbf{a}$ zu 16\%, $5 \mathbf{b ~ z u ~ 3 \%}$ und 5 c zu $38 \%$ enthalogeniert. Da 5 b ein klares Minimum an Enthalogenierung aufweist, muß der zum Enthalogenierungsprodukt führende Mechanismus für la, $\mathbf{5 a}$ ein anderer sein als für $\mathbf{1}$ c, 5c. In untergeordnetem Maße wurde Enthalogenierung von Fluorbenzolen bei der Reaktion von 3-Fluoranisol mit Phenyllithium ${ }^{14)}$ sowie von Fluorbenzol mit tert-Butyllithium ${ }^{15)}$ gefunden und ohne nähere Diskussion über Metall-Halogen-Austausch gedeutet. Für die Enthalogenierung bei 1a, 5a erscheint uns dagegen Hydridübertragung vom Cyclopropyl-Anion an primär gebildetes Arin in Konkurrenz zur normalen nucleophilen Addition des Anions wahrscheinlicher - eine mechanistische Alternative, die schon früher bei Reaktionen von Butyllithium mit Halogenbenzolen in Betracht gezogen wurde ${ }^{16}$. Bricht man die Reaktion von 5 a und 2 statt mit $\mathrm{H}_{2} \mathrm{O}$ mit $\mathrm{D}_{2} \mathrm{O}$ ab, so zeigt die GC-MS-Analyse des Produktgemischs für das gebildete Cyclopropylbenzol (3) keinen Deuteriumgehalt; der $\mathrm{M}+1$-Peak entspricht nahezu exakt dem ${ }^{13} \mathrm{C}$-Isotopenverhältnis für $\mathrm{C}_{9} \mathrm{H}_{10}$. Enthalogenierung über Metall-Halogen-Austausch dagegen lieferte ein in eincr Arylposition vollständig deuteriertes Produkt; eben dieser Befund ergibt sich für 3, das bei der Umsetzung von 5c mit 2 entsteht. Damit ist für die Fluorverbindungen 1a, 5a Enthalogenierung durch Hydridübertragung auf primär gebildetes Arin gesichert. Auf mögliche Folgereaktionen des hierbei gebildeten Cyclopropens gehen wir weiter unten ein.

Chlorbenzole gehen Metall-Halogen-Austauschreaktionen nur ungern ein ${ }^{11}$, aber auch die $o$-Metallierung ist im Vergleich zu den Fluorverbindungen stark verlangsamt. $5 \mathbf{a}$ und 2 ergeben nach $1,2 \mathrm{~h}$ eine Gesamtausbeute an 6 und 7 von $30 \%$, bei $5 \mathrm{~b}$ erhält man nur $6 \%$; die Ausbeute ar enthalogeniertem Produkt sinkt parallel dazu von $16 \%$ bei $5 \mathbf{a}$ auf $3 \%$ bei 5b. Da 6 und 7 aus 5a, b mit Sicherheit über einen Arinmechanismus gebildet werden (s. u.), legt diese auch quantitativ vergleichbare Abnahme den Schlul3 nahe, daß $5 \mathbf{b}$ analog zu 5a weitgehend über die Arinzwischenstufe durch Hydridübertragung enthalogeniert wird.

13) D. E. Applequist und D. F. O'Brien, J. Amer, chem. Soc. 85, 743 (1963).

14) R. Huisgen und H. Rist, Liebigs Ann. Chem. 594, 137 (1955).

15) $R$. Huisgen und L. Zirngibl, Chem. Ber. 91, 1438 (1958).

16) V. Franzen und H.J. Joschek, Angew. Chem. 72, 564 (1960). 
Arinreaktion - Direktsubstitution: Für Cyclopropylsubstituenten ist analog zum Einfluß von Alkylresten ${ }^{11)}$ eine induktive Destabilisierung von Phenyl-Anionen und damit bei zunehmender Cyclopropylsubstitution erschwerte Metallierung und Arinbildung zu erwarten. Andererseits besitzen Cyclopropylreste unbesetzte MOs, die mit dem aromatischen $\pi$-System in Wechselwirkung treten und negative Ladungen stabilisieren können ${ }^{17)}$. Die bei der Direktsubstitution auftretende anionische Zwischenstufe (,anionischer $\sigma$-Komplex") sollte daher durch Cyclopropyl- im Gegensatz zu Alkylgruppen begünstigt und der nucleophile Angriff somit erleichtert werden. Im folgenden wollen wir untersuchen, inwieweit qualitative Voraussagen über die Produktbildung aufgrund dieser Vorstellungen durch die experimentellen Befunde gestützt werden.

Bei den Halogencyclopropylbenzolen 5 ist erschwerte Arinreaktion und begünstigte Direktsubstitution zu erwarten. Die Bildung von 6 und 7 , ausgehend von $5 \mathbf{a}$ bzw. b, erfolgt jedoch sicher über das Arin (Weg A), da beide Produkte in annähernd statistischem Verhältnis entstehen. Beim Abbruch der Umsetzung von 5a und 2 mit $\mathrm{D}_{2} \mathrm{O}$ weist die GC-MS-Analyse ${ }^{18)}$ für $m$ - wie $p$-Dicyclopropylbenzol Molekül-Ionen bei $m / e 159$ aus gegenüber $m / e 158$ bei Aufarbeitung mit $\mathrm{H}_{2} \mathrm{O}$. Fbenso erscheint das für Alkylbenzole charakteristische Tropyliumfragment um eine Massenzahl verschoben bei $m / e$ 92. Der quantitative Einbau von Deuterium in den Arylkern der beiden isomeren Dicyclopropylbenzole stellt einen unabhängigen Beweis dafür dar, daß 6 und 7 aus 5 a ïber eine Arinreaktion entstehen.

Dagegen spricht der hohe Anteil von $7(19 \%)$ gegenüber $6(4 \%)$ bei der Reaktion von 5c für überwiegende Direktsubstitution (Weg B). Wie die Umsetzung von 5a lehrt, werden bei der Arrinreaktion $m$ - und $p$-Dicyclopropylbenzol zu etwa gleichen Teilen gebildet. Die experimentell bestimmten $19 \% 7$ sind damit zu etwa $20 \%$ Arinprodukt, $80 \%$ müssen über Direktsubstitution entstanden sein. Bei der Aufarbeitung mit $\mathrm{D}_{2} \mathrm{O}$ wird in das Produkt der Arinreaktion ein Deuterium eingebaut, während das Substitutionsprodukt D-frei bleibt. Die Intensität des Signals bei $(M+1)$ Masseneinheiten sollte daher $25 \%$ von der des Molekülionensignals betragen, wozu sich aufgrund der ${ }^{13} \mathrm{C}$-Isotopen-Häufigkeit für $\mathrm{C}_{12} \mathrm{H}_{14}$ noch $13 \%$ addieren. Die so errechnete Intensität des $\mathrm{M}+1$-Peaks von $38 \%$ des Molekül-Peaks stimmt in Anbetracht der gaschromatographisch ermittelten Prozentzahlen ganz ausgezeichnet mit dem experimentellen Wert von $32 \%$ überein; die Zuordnung wird auch durch die relative Intensität der Arylfragmente bei $m / e 91$ bzw. 92 und 77 bzw. 78 bestätigt.

Statt durch direkte nucleophile Substitution könnte der Überschuß an 7 auch durch Addition von im Gleichgewicht gebildetem Aryllithium an Cyclopropen entstehen, das entweder bei einer Hydridübertragung oder nach folgendem Mechanismus gebildet wird:

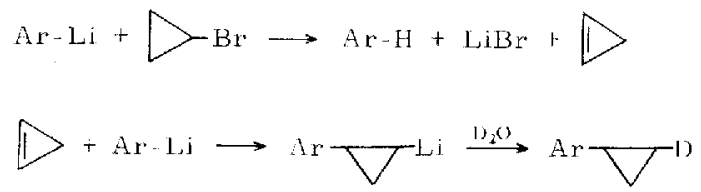

17) J. A. Landgrebe und J. D. Shoemaker, J. Amer. chem. Soc. 89, 4465 (1967).

18) Für die GC-MS-Aufnahmen der Deuterium-markicrten Produktgemische danken wir Dr. M. Seidel von der Badischen Anilin- \& Sodafabrik sehr herzlich, 
Das Schema zeigt, daß hier beim Abbruch mit $\mathrm{D}_{2} \mathrm{O}$ wie bei der Arinreaktion Deuterium in das Molekül eingebaut wird; anhand des Massenspektrums, das D-Gehalt nur für das Produkt der Arinreaktion ausweist, ist dieser Weg daher auszuschließen. Wie Magid und Welch ${ }^{19)}$ berichten, werden bei der Umsetzung von Phenyllithium mit überschüssigem Cyclopropen zudem nur $2.5 \%$ Cyclopropylbenzol gebildet. Wir arbeiten im offenen System in siedendem $\ddot{A}$ ther und können daher Cyclopropanierung auf diesem Weg nicht in nennenswertem Umfang erwarten.

Dieser Versuch beweist, daß bei der Reaktion von $\mathbf{5 c}$ mit 2 der Großteil an $p$-Dicyclopropylbenzol über eine direkte nucleophile Substitution entsteht. Auch das Biphenyl 8 kann hierbei nur durch Direktsubstitution gebildet werden (Weg $\mathrm{C}, \mathrm{R}=$ Cyclopropyl), da bei einer Arinreaktion (Weg D) auch 3,4'-Dicyclopropylbiphenyl nachweisbar sein müßte. Einen weiteren Beweis für die Begünstigung der Direktsubstitution bei der Bromverbindung stellt die Umsetzung von 5c mit Lithium dar, bei der man neben Enthalogenierungsprodukt ( $83 \% 3$ ) nur 10\% 4,4'-Dicyclopropylbiphenyl (8) isoliert, während Reaktion über eine Arinzwischenstufe 8 und das 3,4'-Isomere zu etwa gleichen Teilen liefern müßte. Da sich bei der Reaktion von $\mathbf{5 a - c}$ nur zwei Tricyclopropylbiphenyle, 9 und 10, nachweisen lassen, muß auch hier Direktsubstitution $(\mathrm{D} \rightarrow \mathrm{F} \rightarrow \mathrm{G})$ und nicht Arinreaktion $(\mathrm{D} \rightarrow \mathrm{F} \rightarrow \mathrm{H})$ angenommen werden.

Durch die beiden Dreiringe sollten bei $11 \mathrm{~b}$, c die $o$-Metallierung und, obgleich merklich geringer, auch der Metall-Halogen-Austausch erschwert werden. Trotz vergleichbarer Reaktionszeit ist daher der Gesamtumsatz bei $\mathbf{1 1} \mathbf{b}$ geringer als bei $\mathbf{5 b}$ (26\% zu 8\% Ausgangsprodukt), was sich erwartungsgemäß bei der Arinreaktion $(27 \%$ zu $43 \%$ ) sehr viel stärker niederschlägt als bei der Austauschreaktion $(30 \%$ zu $35 \%$. Die sterische Hinderung durch die $o-\mathrm{C}_{3} \mathrm{H}_{5}$-Gruppe ${ }^{20,4)}$ läßt bei der Addition des Cyclopropyl-Anions an das Arin ausschließlich das Isomere 12 entstehen. Aufgrund dieses sterischen Moments fällt bei 11c auch die nucleophile Substitution, die wegen der besseren Stabilisierung der anionischen Zwischenstufe eher begünstigt sein sollte, wesentlich weniger ins Gewicht als bei 5c: Arinreaktion zu 12 und Direktsubstitution zu 13 laufen in vergleichbarem Umfang ab, und der Anteil der Enthalogenierung hat mit $89 \%$ gegenüber $\mathbf{5 c}$ noch zugenommen.

\section{Struktursicherung}

Zur Identifizierung der Reaktionsprodukte im Produktgemisch wurden aus den analytischen Gaschromatogrammen die Retentionszeiten bestimmt und mit den Werten für authentisches Material verglichen. Teilweise sind von den einzelnen Komponenten der Umsetzungsgemische über eine GC-MS-Kombination noch Massenspektren angefertigt worden.

Die Verbindungen 3, 6, 7 und 12 haben wir auf unabhängigen Wegen dargestellt ${ }^{2}$. Von den Biphenylen lassen sich 4 und das lsomerengemisch 9/10 aus einzelnen Umsetzungen destillativ isolieren, 8 wurde gesondert synthetisiert. 4 weist sich durch das Fehlen von Symmetrieelementen im Arylprotonen-Spektrum eindeutig als 2-Cyclo-

19) R. M. Magid und J. G. Welch, J. Amer. chem. Soc. 90, 5211 (1968).

20) Entsprechend entsteht bei der Umsetzung von 1-Brom-2,4-dicyclopropylbenzol mit $\mathrm{KNH}_{2} / \mathrm{NH}_{3}$ fl ausschließlich 1-Amino-3,5-dicyclopropylbenzol4). 
propylbiphenyl aus, während $8 \mathrm{klar}$ ein $\mathrm{AA}^{\prime} \mathrm{MM}^{\prime}-$ System zeigt. 9/10 sind aufgrund des Intensitätsverhältnisses Arylprotonen: $\alpha-\mathrm{H}: \beta-\mathrm{H}$ von $7: 3: 12$, durch Massenspektrum und Elementaranalyse als Tricyclopropylbiphenyle charakterisiert; die Struktur folgt aus mechanistischen Überlegungen (s. o.). Das bei Umsetzung von 11 c mit 2 gebildete 13 ist nicht isoliert worden, doch zeigt die GC-MS-Analyse zwei Substanzen mit MG 198 und nahezu übereinstimmendem Fragmentierungsmuster. Die Substanz mit der höheren Retentionszeit kann durch Vergleich mit authentischem 122) eindeutig identifiziert werden. Das zweite Isomere, dem wir den unsymmetrischen 1,2,4-Substitulionstyp zuordnen, hat erwartungsgemäß eine kürzere Retentionszeit. Nach dem Reaktionsschema können ausgehend von 11 nur die Tricyclopropylbenzole 12 und 13 gebildet werden, so daß die Struktur von 13 damit ebenfalls als gesichert gelten kann.

Wir danken Frau G. Koller für die sorgfältige Aufnahme der Gaschromatogramme und Herrn W. Blum von der Finnigan GmbH, München, für die kombinierten GC-MS-Analysen. Weiter gilt unser Dank der Deutschen Forschungsgemeinschaft und dem Fonds der Chemischen Industrie für die großzügige Förderung dieser Arbeit.

\section{Experimenteller Teil}

Die analytisch-gaschromatographische Trennung und die Bestimmung der Retentionszeiten von Versuchs- und authentischem Vergleichsmaterial wurden mit einem Varian-Gaschromatographen Modell 1200 über eine 1.7-m-SE 30-Säule durchgefühtt (Integration mit Varian-Integrator Modell 477). Für die Massenspektren wurden die Produktgemische über eine 20-mApiezon L-Stahlkapillare (ID $0.2 \mathrm{~mm}$ ) aufgetrennt und direkt in den Massenspektrographen Finnigan 3000 eingespritzt (Ionisierungsenergie $70 \mathrm{eV}$, Massenbereich $1 \cdots 500$, Empfindlichkeit $107 \mathrm{~A} / \mathrm{V}$ ). Dic ${ }^{1} \mathrm{H}-\mathrm{NMR}$-Spektren wurden mit eincm Varian A-60 gemessen (TMS int. Standard).

a) Darstellung von Cyclopropyllithium (2) (im wesentlichen analog zum Verfahren von Seyferth und Cohen $\left.{ }^{6}\right)$ ): In einem 100-ml-Dreihalskolben mit lnnenthermometer und Magnetrührer werden unter Reinststickstoff zu $1.3 \mathrm{~g}(0.187 \mathrm{~mol})$ feingeschnittenem Lithiumband in $30 \mathrm{ml}$ Äther $9.7 \mathrm{~g}(0.08$ mol) Cyclopropylbromid 21$)$ in $30 \mathrm{ml}$ Ather unter Kühlen so getropft, daß die Innentemp. auf $-1^{\circ}$ bis $+1^{\circ}$ gehalten werden kann ( 90 min). Man rührt 1 h nach. Für weitere Umsetzungen wird die trübc Lösung mit Reinststickstoff über eine G 3-Fritte direkt in das Reaktionsgefäß gedrückt.

b) Allgemeines Verfahren zur Umsetzung von Halogenbenzolen mit $2: \mathrm{Zu}$ der nach a) vorgelegten Cyclopropyllithium-Lösung werden unter Reinststickstoff $0.02 \mathrm{~mol} \mathrm{Halogenbenzol}$ getropft. Es wird unter Rühren und Rückfluß erhitzt (Reakt.-Zeiten s. Tab. 1), wobei sich das Reaktionsgemisch rötlich-braun färbt; teilweise fällt farbloses L,ithiumhalogenid aus. Dann wird abgekühlt und vorsichtig hydrolysiert. Die Ätherphase wird abgetrennt, gewaschen, übcr $\mathrm{MgSO}_{4}$ getrocknet und eingeengt. Der ölige gelbliche Rückstand wird gaschromatographisch analysiert (Tab. 1) bzw. fraktioniert destilliert.

c) 2-Cyclopropylbiphenyl (4): $1.92 \mathrm{~g}(0.02 \mathrm{~mol})$ frisch dest. Fluorbenzol werden, wie unter b) beschrieben, mit 2 umgesctzt. Vom öligen Rückstand $(1.7 \mathrm{~g})$ wird $1 \mathrm{~g}$ fraktioniert destilliert. Als Vorlauf gehen bei $50-54 \% 13$ Torr $0.51 \mathrm{~g} \mathrm{(37 \% )} 3$ über, $n_{\mathrm{D}}^{20} 1.5291$ (Lit.22): $n_{\mathrm{D}}^{26} 1.5306$ ). Ausb, $0.28 \mathrm{~g}(25 \%) 4$, Sdp. $80 \% 0.001$ Torr, $n_{\mathrm{D}}^{20} 1.5953$.

$$
\mathrm{C}_{15} \mathrm{H}_{14} \text { (194.3) Ber. C. } 92.74 \text { H } 7.26 \text { Get. C } 92.83 \text { H } 7.33
$$

21) J.S. Meek und D.T. Osuga, Org. Syntheses 43, 9 (1964). 
NMR $\left(\mathrm{CCl}_{4}\right): \mathrm{H}^{\mathrm{ar}}$ Multiplett $(\mathrm{m})$ zwischen $\delta 6.72$ und $7.62 \mathrm{ppm}(9 \mathrm{H}), \alpha-\mathrm{H} \mathrm{m} 1.55-2.12$ (1 H), $8-\mathrm{H}$ m $0.4-1.03(4 \mathrm{H})$.

d) 4,4'-Dicyclopropylbiphenyl (8): $5.91 \mathrm{~g}(0.03 \mathrm{~mol})$ 1-Brom-4-cyclopropylbenzol 23) (5c) und $0.49 \mathrm{~g}(0.07 \mathrm{~mol})$ Lithium werden in $35 \mathrm{ml}$ Äther unter Reinststickstoff gerührt und zum Rückfluß erhitzt, bis nach $4 \mathrm{~h}$ fast alles Lithium reagiert hat. Das Reaktionsgemisch wird abgekühlt und vorsichtig hydrolysiert, die $\ddot{A}$ therphase abgetrennt, über $\mathrm{MgSO}_{4}$ getrocknet und i. Vak. eingedampft. Der rötliche ölige Rückstand ( $3.3 \mathrm{~g})$ enthält nach dem Gaschromatogramm neben $10 \% 883 \%$ Cyclopropylbenzol, das bei $10^{-3}$ Torr abdestilliert. Der Rückstand wird i. Vak. weiter erhitzt. Bei Ölbadtemp. $180-200^{\circ}$ erstarrt 8 dabei als gelber Feststoff im Kühler, der vorsichtig herausgeschmolzen und aus wenig Äther umkristallisiert wird. Ausb. $0.225 \mathrm{~g}(6.5 \%)$, Schmp. $125-126^{\circ}$, gelblich glänzende Blättchen.

$$
\mathrm{C}_{18} \mathrm{H}_{18} \text { (234.3) Ber. C } 92.26 \mathrm{H} 7.74 \text { Gef. C } 92.15 \text { H } 7.93
$$

NMR $\left(\mathrm{CCl}_{4}\right): 2-, 2^{\prime}-, 6-, 6^{\prime}-\mathrm{H} \delta 7.37 \mathrm{ppm}(4 \mathrm{H}), 3-, 3^{\prime}-, 5-, 5^{\prime}-\mathrm{H} 7.07(4 \mathrm{H}), \alpha-\mathrm{H} \mathrm{m}$ $1.55-2.15(2 \mathrm{H}), 3-\mathrm{H} \mathrm{m} 0.47-1.2(8 \mathrm{H})$.

e) Tricyclopropylbiphenyle $9+10: 2.72 \mathrm{~g}(0.02 \mathrm{~mol})$ frisch dest. 5a werden, wic unter b) beschrieben, mit 2 umgesetzt; man isoliert $2.6 \mathrm{~g}$ Rohöl. Bei der fraktionierten Destillation gehen als Vorlauf $0.66 \mathrm{~g}(28 \%) 3$ über (Sdp. $51-54 \% 13$ Torr, $n_{\mathrm{D}}^{20} 1.5299$ ), bei $108 \cdots 115^{\circ} / 13$ Torr destillicren $1.05 \mathrm{~g}(33 \%)$ Isomerengemisc h $6+7$. Ausb. $0.22 \mathrm{~g}(8 \%)$ Isomerengemisch $9+10$, Sdp. $150-152 \% 0.001$ Torr, zähviskoses gelbliches Öl.

$$
\mathrm{C}_{12} \mathrm{H}_{22} \text { (274.4) Ber. C } 91.92 \text { H } 8.08 \text { Gef. C } 91.70 \text { H } 7.97
$$

NMR $\left(\mathrm{CCl}_{4}\right): \mathrm{H}^{\mathrm{ar}} \mathrm{m} \delta 6.49-7.50 \mathrm{ppm}(7 \mathrm{H}), \alpha-\mathrm{H} \mathrm{m} 1.53-2.17(3 \mathrm{H}), 3-\mathrm{I} \mathrm{m} 0.40-1.13$ $(12 \mathrm{H})$.

f) Umsetzung von Phenyllithium mit Cyclopropylbromid: $3.63 \mathrm{~g}(0.03 \mathrm{~mol})$ Cyclopropy]bromid und $37 \mathrm{ml}(0.03 \mathrm{~mol}) 0.81 \mathrm{~N}$ äther. Phenyllithium-Lösung werden unter Reinststickstoff $12 \mathrm{~h}$ unter Rückfluß gekocht. Gegen Ende der Reaktion fällt cin weißer Niederschlag aus. Nach Abkühlen wird mit Wasser hydrolysiert, die äther. Phase abgetrennt und über $\mathrm{MgSO}_{4}$ getrocknet. Beim Abzichen des Lösungsmittels bleiben 2.9 g rotes Öl zurück, in dem sich gaschromatographisch neben $18 \%$ Biphenyl und weiteren schon in der Phenyllithium-Lösung enthaltenen Verunreinigungen $30 \% 3$ nachwcisen lassen.

In einem zweiten Versuch werden $74 \mathrm{ml}(0.06 \mathrm{~mol}) 0.81 \mathrm{~N}$ äther. Phenyllithium-Lösung und $1.81 \mathrm{~g}(0.015 \mathrm{~mol})$ Cyclopropylbromid wie beschrieben umgesetzt. Man erhält $3.9 \mathrm{~g}$ Rohöl mit $21 \% 3$ (46\%, bez. auf eingesetztes Cyclopropylbromid) und $40 \%$ Biphenyl.

22) T. F. Corbin, R. C. Hahn und H. Shechter, Org. Syntheses 44, 30 (1964).

23) J. Smekal, J. Jonas und J. Farkas, Collect. czechoslov. chem. Commun. 29, 2952 (1964), 